\title{
Estágio clínico em saúde mental: Desconstrução do preconceito ao estigmatizado e construção do cuidado
}

\author{
Clinical internship in mental health: Deconstruction of prejudice to the stigmatized and \\ construction of care
}

Pasantía clínica en salud mental: Deconstrucción del prejuicio al estigmatizado y construcción del cuidado

Recebido: 03/07/2021 | Revisado: 09/07/2021 | Aceito: 10/07/2021 | Publicado: 21/07/2021

\author{
Danton Matheus de Souza \\ ORCID: https://orcid.org/0000-0001-6320-4826 \\ Universidade de São Paulo, Brasil \\ E-mail: Danton_souza@usp.br \\ Gabriella de Andrade Boska \\ ORCID: https://orcid.org/0000-0002-5827-6486 \\ Universidade de São Paulo, Brasil. \\ E-mail: gabriellaboska@usp.br \\ Paula Hayasi Pinho \\ ORCID: https://orcid.org/0000-0001-8922-0699 \\ Universidade Federal do Recôncavo da Bahia, Brasil \\ E-mail: paulahpinho@gmail.com \\ Márcia Aparecida Ferreira de Oliveira \\ ORCID: https://orcid.org/0000-0002-1069-8700 \\ Universidade de São Paulo, Brasil \\ E-mail: marciaap@usp.br
}

\begin{abstract}
Resumo
Objetivo: Relatar a experiência de um estudante de enfermagem acerca da desconstrução do preconceito em relação ao estigmatizado no processo de cuidar em saúde mental a partir da prática clínica da graduação. Metodologia: Estudo descritivo, do tipo relato de experiência, com dados extraídos de um diário de campo de 16 dias composto por reflexões diárias, referentes à experiência do discente de enfermagem no campo prático em saúde mental. Essas reflexões foram analisadas com base no modelo teórico sobre estigma de Erving Goffman e apresentadas de forma descritiva. Resultados: $\mathrm{O}$ estudante chegou ao campo prático com crenças e valores preconceituosos frente às pessoas com problemas de saúde mental, porém, com o desenvolvimento da relação terapêutica permeada pelo vínculo e confiança de ambos os envolvidos, além de automotivação gerada com o cuidado, estudante e paciente, em conjunto, foram capazes de desconstruir e ressignificar suas concepções prévias. Considerações finais: A experiência de inserção no campo prático em saúde mental, somada às possibilidades da relação terapêutica, possibilitaram a desconstrução do preconceito pelo estudante de enfermagem ao indivíduo estigmatizado, com problemas de saúde mental. Além disso, pode reconhecer a integralidade da disciplina frente ao processo de trabalho do enfermeiro.
\end{abstract}

Palavras-chave: Enfermagem; Saúde mental; Estágio clínico; Estigma social; Formação profissional; Ensino.

\begin{abstract}
Objective: To report the experience of a nursing student about the deconstruction of prejudice in relation to the stigmatized in the process of mental health care from the clinical practice of graduation. Methodology: Descriptive study, of the experience report type, with data extracted from a 16-day field diary composed of daily reflections, referring to the experience of the nursing student in the practical field of mental health. These reflections were analyzed based on Erving Goffman's theoretical model on stigma and presented in a descriptive way. Results: The student arrived in the practical field with prejudiced beliefs and values towards people with mental health problems, however, with the development of the therapeutic relationship permeated by the bond and trust of both involved, in addition to self-motivation generated with the care, student and together, they were able to deconstruct and reframe their previous conceptions. Final considerations: The experience of insertion in the practical field of mental health, added to the possibilities of the therapeutic relationship, enabled the deconstruction of prejudice by the nursing student towards the stigmatized individual with mental health problems. Furthermore, it can recognize the integrality of the discipline in relation to the nurse's work process.
\end{abstract}

Keywords: Nursing; Mental health; Clinical clerkship; Social stigma; Professional training; Teaching. 


\begin{abstract}
Resumen
Objetivo: Informar la experiencia de un estudiante de enfermería sobre la deconstrucción del prejuicio en relación a los estigmatizados en el proceso de atención en salud mental desde la práctica clínica de egreso. Metodología: Estudio descriptivo, del tipo relato de experiencia, con datos extraídos de un diario de campo de 16 días compuesto por reflexiones diarias, referentes a la experiencia del estudiante de enfermería en el campo práctico de la salud mental. Estas reflexiones se analizaron con base en el modelo teórico de Erving Goffman sobre el estigma y se presentaron de forma descriptiva. Resultados: El alumno llegó al campo práctico con creencias y valores prejuiciados hacia las personas con problemas de salud mental, sin embargo, con el desarrollo de la relación terapéutica permeado por el vínculo y la confianza de ambos involucrados, además de la automotivación generada con el cuidado, estudiante y juntos, fueron capaces de deconstruir y replantear sus concepciones anteriores. Consideraciones finales: La experiencia de inserción en el campo práctico de la salud mental, sumada a las posibilidades de la relación terapéutica, posibilitó la deconstrucción del prejuicio del estudiante de enfermería hacia el individuo estigmatizado con problemas de salud mental. Además, puede reconocer la integralidad de la disciplina en relación con el proceso de trabajo del enfermero.
\end{abstract}

Palabras clave: Enfermería; Salud mental; Prácticas clínicas; Estigma social; Capacitación profesional; Enseñanza.

\title{
1. Introdução
}

Estigma, de acordo com Erving Goffman, é a posse de uma característica negativa que desacredita e segrega o indivíduo da sociedade. Manifesta-se perante estereótipos, preconceitos e atitudes. Os estereótipos são crenças amplamente aceitas a respeito de hábitos, comportamentos e características atribuídos a indivíduos estigmatizados, que geram respostas emocionais tidas como preconceitos, e também respostas afetivas que levam as atitudes (Goffman, 1963; Phelan, Link \& Dovidio, 2013).

O indivíduo estigmatizado poderia ser facilmente recebido na relação social cotidiana, mas por possuir um traço que pode impor a atenção e desaprovação é excluído e/ou se afasta das relações sociais e de si mesmo (Ribeiro, et. al.,2020; Santos, Barros \& Santos, 2016), o que o torna uma pessoa desacreditada frente a um mundo não receptivo (Goffman, 1963). Isso ocorre em virtude de normas não cumpridas, que geram estereótipos que reforçam a exclusão e a marginalização social (Goffman, 1963; Graham et. al., 2007).

As pessoas com problemas de saúde mental são classificadas por Goffman (1963) no tipo de estigma de culpa de caráter individual, no qual são responsabilizadas por suas características. Nesse sentido, pertencem a um percurso histórico de exclusão social e crenças de que são indivíduos agressivos, perigosos e incapazes (Freitas \& Kebber, 2013; Neto, Silva, Figueira \& Souza, 2016; Souza \& Medrado, 2021), dentre outras concepções que até os dias atuais dificultam sua reabilitação psicossocial e inserção na vida (Weber \& Juruena, 2017; Santos, Barros \& Santos, 2016; Vargas, Maciel, Bittencourt, Lenate \& Pereira, 2018). Essa concepção exerce influência nos modelos de atenção em saúde mental e na atuação prática dos profissionais de saúde, como na enfermagem, área que assumiu a disciplinarização, o controle e o isolamento desses indivíduos como base do "cuidado", até o estabelecimento da Reforma Psiquiátrica Brasileira (Neto, Silva, Figueira \& Souza, 2011; Souza \& Medrado, 2021; Weber \& Juruena, 2017).

A reforma proporcionou a mudança do modelo assistencial, a luta pela igualdade de direitos das pessoas com algum problema de saúde mental ou de uso de álcool e outras drogas, e a promoção de um cuidado humanizado que contempla o estímulo da autonomia e a produção de cidadania (Graham et. al, 2007; Weber \& Juruena, 2017). Contudo, as marcas do estigma e as atitudes preconceituosas ainda permeiam a vivência desse indivíduo em sociedade e até mesmo nos contextos de cuidado, diante da conduta do profissional de saúde (Mersin, Demiralp \& Oksuz, 2019; Rosa, Lima, Miranda \& Peres, 2021).

A adoção de medidas de ensino para aumentar o conhecimento dos estudantes frente a saúde mental se tornou a principal ação indicada para a mudança de crenças, atitudes e práticas excludentes e estigmatizantes em saúde mental (Moreira, 2020; Querido, Tomás, Carvalho, Gomes \& Cordeiro, 2020; Silva \& Marcolan, 2018). Porém, ainda observa-se a baixa abordagem dessa disciplina na formação de futuros profissionais de saúde.

Um estudo brasileiro que avaliou 521 cursos, observou que apenas 41,6\% dos cursos de graduação em enfermagem 
tinham o ensino de saúde mental em sua matriz curricular e 3,4\% não apresentavam nenhum tipo de contato com a área (Vargas, Maciel, Bittencourt, Lenate \& Pereira, 2018), o que reflete na supervalorização da abordagem clínica na formação de futuros profissionais. Outra investigação evidenciou que o foco no físico e no biológico nos cursos de enfermagem tende a formar profissionais que desconsideram aspectos psicossociais, com limitações no uso de tecnologias leves e identificação de necessidades psíquicas das pessoas que cuidam (Mersin, Demiralp \& Oksuz, 2019).

Assim, futuros profissionais ou os já atuantes ao terem contato com a prática clínica em saúde mental podem viver desafios a partir de expectativas negativas, ansiedade, medo e insegurança frente a pessoa em sofrimento psíquico (Freitas \& Kebber, 2013; Rosa, Lima, Miranda \& Peres, 2021; Santos, Barros \& Santos, 2016), sentimentos construídos em sua vivência na sociedade que não lhes deixam livres dos estigmas, e que podem interferir na aprendizagem, refletir na desvalorização da área e em atitudes preconceituosas (Freitas \& Kebber, 2013; Mersin, Demiralp \& Oksuz, 2019). Ao mesmo tempo, a prática assistencial é uma fonte rica de aprendizado para o aluno, bem como, um momento essencial de contato com o sujeito com problemas de saúde mental para a mudança de concepções prévias (Freitas \& Kebber, 2013; Mersin, Demiralp \& Oksuz, 2019; Ramos et. al, 2018).

Dessa forma, este estudo tem como objetivo relatar a experiência de um estudante de enfermagem acerca da desconstrução do preconceito em relação ao estigmatizado no processo de cuidar em saúde mental a partir da prática clínica da graduação.

\section{Metodologia}

Trata-se de um estudo descritivo, de abordagem qualitativa, do tipo relato de experiência, que consistiu em descrever a vivência de um estudante de enfermagem durante sua atuação no campo prático de saúde mental (Pereira, 2018).

O relato foi desenvolvido com base na experiência em um Centro de Reabilitação e Hospital Dia Adulto (CRHD) de um Hospital Psiquiátrico, como parte integrante da disciplina: "Enfermagem em Saúde Mental e Psiquiátrica" de uma universidade pública do estado de São Paulo. O estudante esteve em campo prático durante 16 dias, entre os meses de agosto e setembro de 2019, com um total de 64 horas, e supervisionado por uma docente da universidade. O campo prático (CRHD) tem funcionamento diário e propõe cuidado voluntário às pessoas em sofrimento psíquico e a sua família, integra uma equipe multiprofissional com atividades voltadas ao processo de reabilitação psicossocial.

Como parte integrante da avaliação, os alunos foram orientados a escolher um usuário que frequentasse o serviço e propor a construção conjunta de um Projeto Terapêutico Singular (PTS), com base nas necessidades identificadas. Também foram orientados semanalmente a descrever, de forma livre, a experiência vivida diante do contato com a atuação prática da área de saúde mental, em um relatório entregue semanalmente. Os dados foram coletados por meio desse "diário de campo" em que o discente organizou suas reflexões diárias sobre a experiência, totalizando 16 reflexões.

Os dados foram analisados com base no modelo teórico da obra "Estigma: notas sobre a manipulação da identidade deteriorada", proposta por Erving Goffman (1963) e serão apresentados de forma descritiva, com trechos das reflexões que representaram as desconstruções vivenciadas diariamente pelo estudante durante a experiência prática em saúde mental. As reflexões serão identificadas com a letra $\mathrm{D}$ (dia) seguida de algarismo arábico, que diz respeito ao dia de estágio correspondente à cada reflexão (D1, D2... D16).

O estudo dispensa a aprovação do comitê de ética e pesquisa por se tratar de um relato de experiência produzido pelos próprios autores. 


\section{Resultados}

\subsection{O preconceito diante do indivíduo estigmatizado e início da sua desconstrução}

O estudante de enfermagem iniciou o campo de estágio em saúde mental com uma carga de preconceito que reconheceu como resultado de vivências pessoais e acadêmicas prévias relacionadas a área, o que gerou sentimentos como: medo de agressão física e dos comportamentos das pessoas, insegurança, ansiedade e certo bloqueio a nova experiência, mesmo após toda a carga de conteúdo teórico adquirido previamente ao estágio clínico.

Essas sensações foram nomeadas naquele momento pelo discente de "visão estigmatizante", porém ao analisar a reflexão e perceber que esse conjunto de sentimentos gerou bloqueio ao contato com a pessoa com problemas de saúde mental, é possível afirmar que o estudante ainda mantinha um entendimento marcado de estereótipos e preconceitos, como observa-se nas reflexões seguintes.

“Chego ao estágio com um conjunto de sensações. Medo, insegurança e ansiedade são alguns exemplos. [...] Acredito que ainda mantenho a visão estigmatizante frente a indivíduos em sofrimento mental. [...] Percebi que estava com certo bloqueio, não conseguindo me sentir à vontade no ambiente." (D1)

"Chegar em um estágio com medo, receio, e totalmente desanimado foi uma grande barreira. Medo de ser agredido e de ver surtos. Durante essa fase acadêmica tive contato com pacientes com transtornos mentais, que me deixaram com um certo medo, por gritarem, serem agressivos. [...] Acabei generalizando, o transtorno mental para mim significava pessoas em surto, agressivas e que não tinham nenhum senso crítico.” (D16)

Durante o desenvolvimento da prática clínica, logo no segundo dia, o contato com a pessoa em sofrimento psíquico tornou-se uma oportunidade para a desconstrução da concepção prévia do discente e, a partir das experiências vivenciadas, novas reflexões foram se estruturando. $\mathrm{O}$ estudante teve contato com uma usuária sobre a qual já tinha ouvido relatos da história de sua crise, situação que resultou em internação psiquiátrica associada a um processo judicial, por ter sido comparado a um crime por infração às leis do Estatuto da Criança e do Adolescente. O ocorrido gerou afastamento de sua família e rede de apoio e pela ampla divulgação nos veículos midiáticos, dividiu opiniões sociais e impulsionou um contexto de estigmatização coletiva da usuária, que ocorreu até mesmo dentro do próprio serviço de saúde mental. Esse fato despertou o interesse em compreender as histórias naturais das pessoas com problemas de saúde mental, considerando as experiências que contribuíram para o adoecimento psíquico, não mais definindo essas pessoas como desacreditadas e olhando-as com foco na história natural do estigma.

\footnotetext{
“Após refletir acredito que devo ser mais aberto, deixar todo o passado de lado e estar ali, disponível, e pronto para lidar com o novo desafio. [...] Tentando iniciar uma nova perspectiva sobre a área." (D2)
}

Iniciou assim o desenvolvimento do Relacionamento Interpessoal Terapêutico (RIT) onde o estudante teve a oportunidade de colocar em prática as fases de orientação, identificação, exploração e resolução da Teoria de Peplau (1952) e essas acompanharam o processo de mudança e evolução do processo de cuidar. A fase de orientação ocorreu ao longo de vários dias e, nesse período, o vínculo e a confiança se consagraram como aspectos transformadores das atitudes preconceituosas do estudante, esta etapa foi iniciada pelos próprios usuários do serviço. Conforme trechos abaixo: 
"Ser abordado pelos pacientes me deixa feliz e mais seguro de mim, pois sinto que estou conseguindo conhecê-los e criar vínculo." (D4)

"Os pacientes já me abordam. [...] Sinto que além de criar vínculo com a paciente escolhida, consegui também com outros usuários do serviço." (D5)

Ao longo dos dias, o estudante passou a ser reconhecido como referência para outros usuários, que também apresentavam necessidades de cuidado e com isso se deparou com diversas demandas subjetivas, difíceis de ouvir e abordar, ainda mais para aquele que está vivenciando a primeira experiência em saúde mental, o que gerou no discente esgotamento emocional associado a um desgaste físico. Aos poucos esse foi reconhecendo seus limites e a necessidade de também cuidar da sua própria saúde mental.

"Fui embora com uma intensa dor de cabeça, acho que preciso me preparar mais para aguentar o tanto de demanda que está surgindo de outros pacientes, algumas pesadas.” (D7)

"Sai do estágio com um pouco de dor de cabeça, acho que lidar com tantos problemas me deixa um pouco estressado psicologicamente." (D9)

Com o avançar do RIT o estudante deu início às intervenções de enfermagem e as etapas de Identificação e Exploração na segunda semana do estágio. Essas fases marcaram o momento em que o cuidado deixou de ser desgastante para trazer transformações pessoais com reconhecimento da importância do relacionamento interpessoal, da complexidade do cuidado de enfermagem em saúde mental e das transformações mútuas proporcionadas com esse.

"É interessante como muitas coisas que se vivencia como estagiário nesta área podem ser levadas para a própria vida, desde reflexões, até possíveis ações futuras. [...] O que mais me surpreende é que estou gostando de trabalhar com isso. [...] Ver o retorno positivo dos pacientes me deixa com mais vontade de continuar." (D5)

"Hoje foi extremamente gratificante ouvir a paciente me dizer que estou conseguindo ajudá-la. [...] Ouvi que deveria seguir a área de saúde mental, algo que jamais pensei. [...] É bom perceber uma evolução.” (D6)

\subsection{Ressignificando o cuidado de enfermagem em saúde mental}

$\mathrm{Na}$ última semana de estágio, o estudante iniciou a fase de resolução do RIT. Até esse momento ( $12^{\circ}$ dia em campo) não havia consultado o histórico dos usuários nos respectivos prontuários, visto que esses continham avaliações e anotações de outros profissionais da equipe e reconheceu que a depender do olhar que foi dado por eles aos casos, poderia reforçar atitudes preconceituosas e prejudicar o desenvolvimento da relação terapêutica. Essa escolha foi validada pelo estudante após passar pela experiência.

\footnotetext{
"Interessante deixar para olhar os prontuários nos últimos dias, lemos coisas que não imaginávamos, e que se tivéssemos visto antes teríamos sido influenciados pelo julgamento. [...] Acredito que poderíamos ter uma "pré-visão" dos pacientes, o que atrapalha o vínculo." (D12)
} 
Apropriando-se do cuidado em saúde mental e reconhecendo suas potencialidades, o estágio clínico permitiu a construção conjunta de intervenções de enfermagem que consideraram o outro como um ser humano de histórias, direitos, memórias, afetos, medos e necessidades, para além do diagnóstico psiquiátrico. Percebe-se que ao final desse processo de desconstrução do preconceito o estudante passou a reconhecer seus preconceitos e a importância da mudança pessoal para que o cuidado ocorra.

“Estar com essas pessoas me ajuda a refletir sobre toda a carga preconceituosa que cheguei ao campo de estágio e como isso influenciava a maneira de olhar o outro. O processo de mudança, também se aplica a mim." (D14)

"O receio vinha por acreditar que iria estagiar em meio a contenções, sedações, entre pacientes que iriam me manipular para conseguir o que desejavam. Um grande estigma criado socialmente, que mesmo sendo um futuro profissional de saúde, estava levando isso como verdade absoluta." (D16)

Diante das transformações pessoais e profissionais, o discente reconheceu que independente da especificidade que será seguida, o futuro profissional deve olhar o sujeito em seus aspectos biopsicosocioespiritual, sendo a saúde mental parte fundamental dessa integralidade.

"Acredito que futuros profissionais de saúde que tenham contato com a saúde mental, independente da área que esteja seguindo, utilizarão toda a carga de conhecimento gerado pela teoria e pela prática em sua atuação. [...] Os profissionais precisam estar preparados para cuidar, sem esquecer de nenhum âmbito, independente da área que esteja." (D16)

O campo de prática gerou mudanças positivas e significativas, tanto do ponto de vista pessoal com a mudança de crenças e estereótipos às pessoas com problemas de saúde mental, quanto na vida profissional, com a possibilidade de exercer a teoria, entender a transversalidade da saúde mental com outras disciplinas e reconhecer a importância do estudo e atuação na área. Assim, observa-se que o campo prático se tornou um preditor positivo na desconstrução de preconceito à pessoa com problema de saúde mental, como ilustra a Figura 1, abaixo.

Figura 1 - Experiência prática em Saúde Mental e suas transformações. São Paulo, SP. Brasil, 2019.

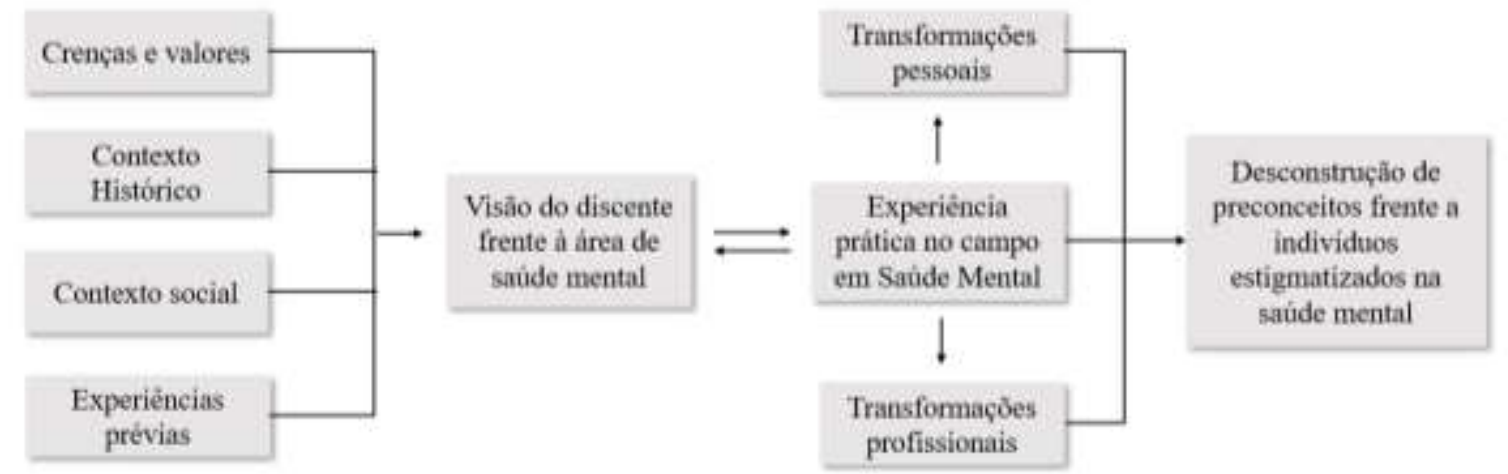

Fonte: Autores. 


\section{Discussão}

Diante das reflexões do estudante de enfermagem ao decorrer do processo de cuidar em saúde mental na prática clínica da graduação, podemos afirmar que mesmo que esse esteja preparado teoricamente para a assistência, não está livre da demonstração de preconceitos e influências das concepções históricas da "loucura". Contudo, a vivência do estágio clínico pode facilitar a desconstrução dos preconceitos frente às pessoas com problemas de saúde mental: às pessoas estigmatizadas.

Goffman (1963) problematiza que: "O normal e o estigmatizado não são pessoas, e sim perspectivas geradas em situações sociais durante os contatos mistos, em virtude de normas não cumpridas que provavelmente atuam sobre o encontro". Nesse sentido, observamos que a perspectiva do discente era de que o encontro com pessoas com problemas de saúde mental seria de normas sociais incumpridas, com preconceitos que reduzem o outro a uma pessoa sem capacidades, com atitudes de desqualificação e descrédito. Porém, as duas primeiras semanas de prática naturalmente desconstruíram essa perspectiva e proporcionaram que o outro fosse acolhido em sua individualidade.

Essa desconstrução se iniciou com o vínculo, e o primeiro contato do discente com a área de saúde mental trouxe à tona estereótipos, com sentimentos semelhantes à de outros discentes de cursos de saúde, identificados em uma revisão de literatura (Freitas \& Kebber, 2013). Porém, o estudante pode reconhecê-los precocemente e, com a automotivação no D2, ou seja, a vontade de aprender mais e ter maior envolvimento com o assunto, se afastou da construção coletiva do estigma e se mostrou aberto à vivência.

Os estereótipos e atitudes preconceituosas influenciam a atuação do profissional e o modo de viver dos indivíduos com problemas de saúde mental (Santos, Barros \& Santos, 2016; Ribeiro, et. al., 2020; Rosa, Lima, Miranda \& Peres, 2021), e para Goffman (1963) os profissionais de saúde devem se afastar dessas visões e ter como foco a individualidade do sujeito, como realizado pelo discente em questão, no qual se disponibilizou ao contato, com comunicação cíclica e reciprocidade entre os envolvidos, que levou à construção do vínculo, medidas essenciais para a construção do cuidado integral e humanizado, com intervenções possíveis e livre de estigmas.

Vale ressaltar que o estudante se vinculou com uma usuária vista com descrédito e desqualificação por símbolos de estigma (Goffman, 1963) como a crise, a internação psiquiátrica, o processo judicial e o "crime”. Observa-se que após a ver como um sujeito de direitos e se motivar a conhecer sua história natural (Goffman, 1963), deixando de lado suas pré concepções, pode iniciar o RIT, ferramenta que influenciou a desconstrução aqui relatada.

Outro ponto a ressaltar é que na saúde mental, a vinculação do profissional com o usuário é base do processo de cuidar (Caldas, et. al., 2021; Martinez C, et. al., 2019; Souza, Boska, Oliveira \& Oliveira, 2021), mas há impasses na assistência quando esse é marcado pela inserção do sujeito dentro de seus estigmas. Quando visões pessoais são postas acima dos princípios da profissão, há uma má qualidade de tratamento, que impactam no acesso aos serviços, cuidados fornecidos e marginalização dos indivíduos (Goffman, 1963; Rosa, Lima, Miranda \& Peres, 2021; Santos, Barros \& Santos, 2016). Porém, neste estudo observa-se que o contato prático se mostrou como um preditor positivo à desconstrução de preconceitos, e esses dados corroboram com investigação realizada com 185 estudantes de enfermagem durante práticas em saúde mental, na qual observou que o contato direto com pessoas com problemas de saúde mental proporcionou transformação nas crenç as e atitudes preconceituosas, reafirmando que o contato se torna parte fundamental do processo de mudança, associado à diminuição de sentimentos de desqualificação do outro, por parte dos estudantes (Martinez, et. al., 2019).

Ressaltamos que o fato do estágio ocorrer em um hospital psiquiátrico, pode ter influenciado nas ideias préconcebidas do discente em relação ao cuidado ser produzido em meio a contenção e sedação, tanto que após 16 dias é que a ressignificação dessas sensações e do cuidado em saúde mental se tornaram concretas, fator que pode estar diretamente relacionado ao tempo de inserção no cotidiano dos serviços.

Goffman traz como reflexão que, para alterar a realidade de estigmas, não devemos olhar para a diferença, mas sim 
para o comum (Goffman, 1963). Inicialmente o comum parecia não existir e resquícios das práticas da enfermagem psiquiátrica manicomial apareceram nas reflexões do estudante. A partir do D5 percebemos relatos de que os aspectos do cuidado em saúde mental se aplicavam à vivência pessoal do discente, ou seja, o comum estava ali. A relação terapêutica entre enfermeiro-paciente quando estabelecida promove crescimento e envolvimento mútuo e, frequentemente, os mesmos problemas, medos, angústias expressadas pelos usuários são vivenciados pelos profissionais (Peplau, 1952; Rosa, Lima, Miranda \& Peres, 2021; Souza, Boska, Oliveira \& Oliveira, 2021).

Quando o enfermeiro acessa esses sentimentos, mesmo que indiretamente, depara-se com situações de desgaste físico e emocional, como referido pelo estudante no D7 e no D9. O aparecimento dessa situação estressora apresenta correlação positiva com o processo de ensino e aprendizagem, e no caso da experiência em saúde mental, para além da dimensão clínico assistencial, o autoconhecimento (Peplau, 1952; Souza, Boska, Oliveira \& Oliveira, 2021).

No contexto da saúde mental, além de intervir no normal, também deve-se ter o cuidado com o próprio indivíduo estigmatizado, uma vez que o diagnóstico psiquiátrico, na prática clínica, aparece muitas vezes como limitador do cuidado por focar no rótulo da "doença" sendo intrinsecamente um fator de auto estigmatização para os sujeitos. Goffman aborda que o estigma carregado por indivíduos com diagnóstico de transtorno mental, demonstrado pelo meio social, pode ser tido por ele como uma identidade. A pessoa estigmatizada aprende e incorpora o ponto de vista dos "normais" que a tratam como desacreditada, adquirindo as visões da sociedade mais ampla em relação a uma ideia geral do que significa possuir um estigma particular, e as consequências de possuí-lo. Assim, os próprios indivíduos com essas questões, não diferenciam a história natural da "loucura" com a sua biografia, limitando-se ao seu diagnóstico psiquiátrico, reproduzindo a visão e a perspectiva social desse, não se reconhecendo como um ser humano digno de um destino agradável e uma oportunidade legítima, assumindo o lugar de desacreditável (Goffman, 1963).

Assim como todo estigma, a auto estigmatização tem influência histórica (Goffman, 1953; Santos, Barros \& Santos, 2016) e mesmo com a evolução assistencial percebe-se a dificuldade das pessoas em sofrimento mental para a desinstitucionalização, prática da cidadania e de entendimento dos seus direitos humanos (Neto, Silva, Figueira \& Souza, 2021; Ribeiro, et. al, 2020; Weber \& Juruena, 2017). Portanto, faz-se necessário estruturar ações de enfermagem em saúde mental que possibilitem considerar o outro dentro dessa limitação e não reforçar esse lugar. A estratégia do estudante em consultar o diagnóstico e os prontuários após desenvolver o seu próprio entendimento sobre aquelas pessoas pode ter contribuído com essa problemática.

Uma investigação que buscou analisar a percepção de pessoas com problemas de saúde mental sobre o preconceito, encontrou que o estigma vindo do meio familiar e de cuidado à saúde era a causa de maior sofrimento, além de agravar o quadro clínico. Percebe-se que reduzir a carga de estigma e romper com as atitudes e sentimentos preconceituosos favorecem o cuidado e reduz o sofrimento (Silva \& Marcolan, 2018).

Para tal, há a necessidade de promoção do debate, em todos os níveis de atenção sobre o estigma, promoção e proposição de políticas, e adotar medidas de ensino para mudar crenças e atitudes dos profissionais e, também, dos discentes que ainda estão em seu processo de construção e serão os profissionais atuantes no futuro (Graham et. al., 2007), visto que, o despreparo para lidar com seus próprios sentimentos e angústias diante de situações difíceis, fragiliza a formação de enfermeiros autônomos, críticos, reflexivos, empáticos e principalmente humanos.

\section{Considerações Finais}

A experiência de inserção no campo prático em saúde mental durante 16 dias, somado à construção conjunta de um PTS por meio do RIT, possibilitaram a desmistificação de estereótipos, ideias preconcebidas e atitudes preconceituosas pelo estudante de enfermagem ao indivíduo estigmatizado com problemas de saúde mental. Esse encerrou sua vivência clínica sem 
medo do contato com a "loucura", mas com a capacidade de olhar o indivíduo de forma integral, promover o cuidado e estar próximo desse durante o sofrimento. Além disso, pode reconhecer a integralidade da disciplina frente ao processo de trabalho do enfermeiro.

Por trazer o relato de apenas um discente não é possível demonstrar a magnitude do problema, porém, espera-se que este estudo contribua para discussões teórico práticas nos cursos de graduação em enfermagem e outras áreas da saúde, em relação ao conceito do estigma e sua estruturação, como também, a importância do estágio clínico em saúde mental na formação de futuros profissionais.

Constata-se a necessidade de novas pesquisas na área, que identifiquem, em uma população representativa, fatores que influenciam na formação de crenças, estereótipos e preconceitos, e os fatores que auxiliam nessa desconstrução. Além disso, torna-se relevante medidas de ensino que auxiliem o processo de valorização da saúde mental, como parte integrante do cuidado, e ressignificação do preconceito frente ao estigmatizado.

\section{Referências}

Caldas, G. R. F., et. al. (2021) Nursing and Psychiatry- Trajectory and Evolution: A literature Review. Research, Society and Development; 10(2).

Freitas, B. M. C. \& Kebbe, L. M. (2013) Mental Health by the perception of trainees: A literature review. Psicologia Argument. 31(74): 519-28.

Goffman, E. (1963) Estigma- Notas sobre a manipulação da identidade deteriorada. 124p.

Graham, N., et. al. (2007) Reducing stigma and discrimination against older people with mental disorders: a technical consensus statement. Rev. Psiq. Clín. 34(1): 39-49.

Martínez, C., Martínez, V. S., Orts, R. S., Dinca, A., Martínez, M. R. \& Pichardo, J. D. R. (2019) Effectiveness of direct contact intervention with people with mental illness to reduce stigma in nursing students. Int J Ment Health Nurs. 28(3): 735-43.

Mersin, S., Demiralp, M. \& Öksüz, E. (2019) Addressing the psychosocial needs of patients: Challenges for nursing students. Perspect Psychiatr Care. 1-8.

Moreira, M. I. B. (2020) Shared trajectories: experiences of students, users and family members of mental health services in teaching-learning actions in health. Saúde Debate. 44(127): 1189-1200.

Neto, J. B. M., Silva, E. S. M., Figueira, G. M. \& Souza, J. C. (2021) The Stigma of mental illness among students and health professionals. Research, Society and Development. 10(3).

Peplau, H. E. (1952) Interpersonal Relations in Nursing. 1952. 356 p.

Pereira, A. S., et al. (2018). Metodologia da pesquisa científica. UFSM.

Querido, A. I. F., Tomás, C. C., Carvalho, D. R. S., Gomes, J. M. F. \& Cordeiro, M. S. S. (2020) Impact of an intervention on stigma in mental health and intergroup anxiety. ACTA Paul Enferm. 33:1-9.

Ramos, T. K., Nietsche, E. A., Cogo, S. B., Cassenote, L. G., Bock, A. \& Martins, F. S. (2018) Supervised Curricular Internship and training of the Nurse: Developed Activities. Rev Enferm UFSM. 8(1): 59-71.

Ribeiro, A. F. S., et al. (2020) Social stigma, family functioning pattern and the importance of treating mental disorders for users of psychoactive substances. Research, Society and Development. 9(8).

Rosa, D. C. J. R., Lima, D. M., Miranda, L. \& Peres, R. S. (2021) "Paciente-problema": Imaginário coletivo de enfermeiros acerca do usuário com diagnóstico de esquizofrenia.Physis: Rev de Saúde Coletiva. 31(1).

Santos, J. C., Barros, S. \& Santos, I. M. M. (2016) Stigma: The Perspective of Workers on Community Mental Health Services- Brazil. Global Qualitative Nursing Research. 3: 1-9.

Silva, T. C. M. F. \& Marcolan, J. F.(2018) Preventing individuals with mental disorders as a grievance of suffering. Rev Enf. UFPE. 2018; 12(8): 2089-98.

Souza, D. M., Boska, G. A., Oliveira, M. S. R. \& Oliveira, M. A. F. (2021) Construction of mental health care based on the experiences of a nursing student. Rev Bras Enferm. 74(3).

Souza, R. M. \& Medrado, A. C. C. (2021) On bodies as object: a postcolonial reading of the 'Brazilian Holocaust'. Saúde Debate. $45(128)$ : 164-177.

Phelan, J., Link, B. \& Dovidio, J. (2013) Estigma e preconceito: Um animal ou dois? Estigma e Saúde. 2013; $183-207$.

Vargas, D., Maciel, M. E. D., Bittencourt, M. N., Lenate, J. S. \& Pereira, C. F. (2018) O Ensino de Enfermagem Psiquiátrica e Saúde Mental no Brasil: Análise Curricular da Graduação. Texto \& Contexto Enferm. 27(2): 1-9.

Weber, C. A. T. \& Juruena, M. F. (2017) Paradigm in care and stigma of the mental disorder in Brazilian psychiatric reform. Psicologia, Saúde e Doença. 18(3): 640-56. 